\title{
Linx
}

Revue des linguistes de l'université Paris X Nanterre

39 | 1998

Modèles linguistiques : convergences, divergences

\section{Identification de deux constructions en comme : causalité et comparaison}

Identification of two constructions with comme in French: causality and comparison

\section{Marianne Desmets}

\section{OpenEdition}

\section{Journals}

Édition électronique

URL : http://journals.openedition.org/linx/879

DOI : $10.4000 /$ linx. 879

ISSN : 2118-9692

\section{Éditeur}

Presses universitaires de Paris Nanterre

Édition imprimée

Date de publication : 15 décembre 1998

Pagination : 89-118

ISSN : 0246-8743

Référence électronique

Marianne Desmets, «Identification de deux constructions en comme : causalité et comparaison », Linx [En ligne], 39 | 1998, mis en ligne le 02 juillet 2012, consulté le 19 avril 2019. URL : http:// journals.openedition.org/linx/879; DOI : 10.4000/linx.879

Ce document a été généré automatiquement le 19 avril 2019

Département de Sciences du langage, Université Paris Ouest 


\title{
Identification de deux constructions en comme : causalité et comparaison
}

\author{
Identification of two constructions with comme in French: causality and \\ comparison
}

Marianne Desmets

1 Les constructions en comme représentent une partie de langue peu étudiée pour ellemême. Dans les grammaires classiques, on les trouve regroupées avec d'autres, classées bien souvent selon des critères d'usages. Les emplois de comme sont forts variés, et il est vrai que de tels ouvrages, par ailleurs précieux pour la richesse des données qu'ils fournissent, n'en présentent pas de descriptions systématiques ou unifiées. Comme est également le "parent pauvre» de la linguistique contemporaine, particulièrement en syntaxe. Signalons les travaux de Caron-Pargue (1992) et de Le Goffic (1991) se situant dans le champ des sciences cognitives, en syntaxe l'analyse de Milner (1973), qui traite comme par analogie avec les constructions comparatives en que. Le présent article se situe dans une perspective plus large de modélisation des structures du français dans le cadre d'une grammaire syntagmatique de type HPSG. L'étude élaborée ici constitue la première étape de description nécessaire à toute formalisation d'un phénomène en langue naturelle. C'est aussi, et surtout, l'occasion d'exposer les problèmes d'analyse et de représentation que posent des faits de langue peu étudiés. Nous limitons notre propos aux structures phrastiques, causales et comparatives en comme. Le corpus utilisé est constitué d'exemples attestés (Le Monde et divers textes littéraires) et d'exemples construits qui ont été soumis au jugement de nombreux locuteurs.

2 Nous présentons tout d'abord des critères de distinction formels. Examinons les caractéristiques générales des structures suivantes, causale en (1), comparative en (2). Leur réalisation est assez semblable :

(1) Comme Pierre ne l'avait pas encore fait, il présenta sa femme à son nouvel employeur. 

affaiblissent nos parts de marché.

Selon la terminologie traditionnelle, on a affaire à des constructions subordonnées. Par subordination on entend les deux caractéristiques structurales suivantes: (i) un marqueur initial, ici comme, introduisant un syntagme de type phrastique, la subordonnée; (ii) un élément d'une phrase matrice auquel est adjointe la phrase subordonnée. Or, la description des constructions causales et comparatives en comme impose de discuter ces deux points avec précision. En (1) comme en (2), de quelle catégorie est l'élément auquel s'adjoint la subordonnée ? De quelle nature est le lien qu'ils entretiennent? Enfin, étant donnée la position initiale de la subordonnée dans les énoncés (1) et (2), comment peut-on à la fois rendre compte des propriétés de constituance et des contraintes attachées à l'ordre linéaire des réalisations? Nous verrons que la distinction formelle des réalisations causales et comparatives est fonction d'une combinatoire de contraintes externes (élément modifié par l'ajout en comme et place de l'ajout) et internes (réalisation de l'objet et inversion du sujet).

Dans un second temps, on s'attachera à la description des stratégies linguistiques utilisées dans les constructions comparatives pour le traitement de l'information. Nous étudierons des phénomènes relevant de la copie (copie lexicale et anaphore) et de l'ellipse (ellipse lexicale et dépendance à distance d'un argument).

\section{Distinction de la construction causale et des constructions comparatives}

\subsection{Configuration d'adjonction et relation de modification}

5 Suivant le patron structural d'une subordonnée relative ou complétive, on s'attend à ce que figure dans la configuration des constructions en comme un constituant tête de la catégorie maximale ${ }^{2}$, nœud sœur gauche de la subordonnée. Manifestement pour (1) et (2), on ne peut justifier d'aucun constituant interne de la principale (de type N, A ou V) capable de remplir ce rôle. C'est l'ensemble de la phrase matrice qui semble constituer le noeud soeur.

On envisage d'autre part deux types de relations entre la subordonnée et le constituant tête : soit on est dans un cas de sous-catégorisation, soit on est face à une adjonction simple. Si l'hypothèse qui a été formulée plus haut est correcte, nous nous trouvons dans le second cas. S, noeud soeur de la subordonnée, constitue une catégorie maximale qui, au sens strict, ne sous-catégorise pas de complément. Cette configuration implique des caractéristiques syntaxiques et sémantiques. Du point de vue syntaxique, on se situe dans une configuration d'ajout: un syntagme est adjoint à un constituant tête sans que le domaine supérieur alors constitué ne change de catégorie. Du point de vue sémantique, on dira qu'un élément adjoint modifie le contenu d'un autre élément au sens où l'apport informationnel du tout est celui de l'élément tête révisé par celui de l'élément adjoint. 


\subsection{Première distinction : Causale $\mathrm{MOD}^{3} \mathrm{~S} /$ Comparative MOD X}

7 Les causales et comparatives en comme appartiennent à ce que les grammaires traditionnelles appellent « propositions adverbiales » (Grevisse (1961) §1083 et §1085), ou encore « circonstancielles» (Le Bidois (1950) ch. V : 312 et s.v.; Sandfeld (1936) §196 et s.v., §256 et s.v.), ce qui confirme leur statut d'ajout. Pourtant, les réalisations ne sont pas uniformes. En (1) et (2) on peut avancer que l'ensemble de la phrase subordonnée a comme portée le cadre de la phrase principale, non pas un de ses constituants. Si ce cas est général à toute réalisation de comme causal (exemples (3)-(4)), les constructions en comme comparatif laissent apparaître d'autres types de modification que celui du S principal (exemples (5)-(15)).

\subsubsection{Subordonnée causale, MOD S}

(3) Comme les chevaux ne sont pas encore attelés, Tartarin propose négligemment d'aller faire un tour jusque-là.

(4) Pierre regardait son père et sa mère, mais comme on parla d'autre chose, il se remit à boire. (Sandfeld \$196)

\subsubsection{Subordonnée comparative, MOD X}

\section{Modification de S}

(5) Même si la France, comme l'affirme le Quai d'Orsay, n'a pas de réserves à exprimer sur cette attaque d'un site nucléaire à Bagdad, il serait bon de se soucier de la population.

\section{Modification du GV principal}

(6) Il vous a traité comme il aurait traité son fils. (Grevisse § 1085)

(7) Je songe à des moulins beaux, forts et vigoureux, comme y songerait un enfant captivé par le vent.

\section{Modification d'un GV infinitif (GVinf autre que le GV principal)}

(8) L'ambition générale est de contrer toute velléité de l'Irak de revenir sur cette limitation de sa souveraineté, comme entend le faire le régime de Bagdad en posant des conditions aux avions des inspecteurs du désarmement de l'ONU qui survoleraient cette zone d'exclusion. (Le Monde)

\section{Modification d'un GN}

(9) Je rêve à des gâteaux au chocolat comme ma nounou les préparait. 
(10) La "maison du peuple ", comme l'avait baptisée George Bush le jour de son investiture, le 20 janvier 1989, a subi ses derniers grands travaux sous la présidence du démocrate John Kennedy.

(11) D'autre part, vous négligez la dimension historique, la "dignité du temps", comme disait Mme de Staël, qui seule dessine l'évidence des progrès et minimise la portée des accidents de parcours.

Modification d'un A ou GA

\begin{tabular}{|l|l|}
\hline (12) & Malade comme était la corvette, elle était peu résistante aux secousses. (Grevisse \$1085) \\
\hline (13) & Il s'en va bon train, heureux comme il est d'avoir réussi son examen. \\
\hline (14) & Il est fier comme est fier un père venant de marier sa fille. \\
\hline (15) & Il est très fier de ses ancêtres comme peut l'être un fils de notable. \\
\hline
\end{tabular}

8 L'analyse du constituant modifié repose sur l'interprétation sémantique et détermine le lieu de l'adjonction. En ce qui concerne les constructions causales, on ne rencontre pas de difficultés: le schéma interprétatif est constant, la catégorie modifiée et la place antéposée qu'elles occupent le sont aussi. Les comparatives, par contre, présentent une variété de réalisations. Nous pensons ${ }^{4}$ qu'une répartition a priori en termes d'usage " circonstanciel de manière ", « comparatif», ou « incidente de discours", ne rendrait pas compte du sens central de la construction comparative, ni surtout de l'unité des contraintes en jeu. Notre hypothèse de départ est qu'il n'y a que deux grands types de réalisations comme- $\mathrm{S}^{5}$ : les formes causales et les formes comparatives. Conformément à Goldberg (1995) ${ }^{6}$ et à Sag (1996), nous avançons que l'étude des contraintes syntaxiques et sémantiques de ces constructions doit nous permettre de dresser une typologie de leurs réalisations particulières. Les paramètres de variation existent en nombre fini, il s'agit au niveau des propriétés externes, de la modification et des possibilités de déplacement de la subordonnée; au niveau des propriétés internes, des relations d'anaphore et de la réalisation linéaire des constituants de la subordonnée. Ces contraintes sont cooccurrentes, en étudiant leurs combinatoires nous mettons à jour les traits propres à chacune des réalisations en comme.

\subsection{Deuxième distinction : la place de la subordonnée en comme}

9 Dire que ces deux types de construction ont une configuration d'ajout ne suffit pas à décrire l'ensemble de leurs propriétés structurales, puisque dans certains cas ces ajouts phrastiques sont déplaçables -- propriété assez générale des ajouts "circonstanciels", même si elle se manifeste avec de nombreuses contraintes (selon la classe d'adverbe, le type de GP, etc.). Les modèles grammaticaux contemporains (théorie générativiste ou modèles plus récents de type grammaires d'unification) ne développent pas toujours les outils nécessaires à la représentation des contraintes sur la réalisation linéaire des constituants. De façon générale, la distinction n'est pas faite entre structure de constituants et réalisation linéaire ${ }^{7}$. Dans les cas qui nous préoccupent, les propriétés de 
déplacement ne déterminent pas l'existence de nouvelles structures, elles sont une caractéristique supplémentaire dont il faut rendre compte.

\section{Place de l'ajout causal}

10 Soit un patron linéaire P1 (place 1), P2 (place 2), censé représenter les places de réalisation linéaire de la subordonnée et de la principale. On observe que la subordonnée causale est toujours en P1, devant la catégorie S qu'elle modifie -- ce que confirment les observations de Grevisse, Sandfeld et Le Bidois (pour ne citer qu'eux).

\begin{tabular}{|l|l|l|}
\hline (16) & a. & Comme il avait faim, il mangea tout aussi vite qu'il but. \\
\hline & b. & ??Il mangea tout aussi vite qu'il but, comme il avait faim. \\
\hline (17) & a. & $\begin{array}{l}\text { Comme il ne voulait à aucun prix se laisser reconnaître, il dut recourir à la ruse pour pénétrer } \\
\text { dans le château. }\end{array}$ \\
\hline & b. & $\begin{array}{l}\text { ??ll dut recourir à la ruse pour pénétrer dans le château, comme il ne voulait à aucun prix se } \\
\text { laisser reconnaître. }\end{array}$ \\
\hline
\end{tabular}

\section{Places des ajouts comparatifs}

11 La distribution des places d'un ajout comparatif en comme se combine à deux autres propriétés : (i) une propriété externe : le type de constituant modifié ; (ii) une propriété interne: la réalisation syntagmatique ou non, de l'objet du verbe de la subordonnée. Selon les formes étudiées, nous regardons les places suivantes: devant/derrière le $\mathrm{S}$ principal (P1/P2) ; devant/derrière le constituant modifié $(\mathrm{C} 1 / \mathrm{C} 2)$; en position incidente à l'intérieur du constituant modifié. Le lieu d'insertion de l'ajout diffère selon les constituants, il se trouve très souvent entre le sujet et le verbe de la principale pour une comparative MOD S, entre le Vinf et ses compléments pour une comparative MOD GVinf, entre le A et ses compléments pour une comparative MOD GA. Il semble, par contre, qu'il n'y ait pas de position d'insertion possible dans un $\mathrm{GN}^{8}$.

\subsubsection{Comparative MOD GV, avec réalisation canonique de l'objet}

Lorsque le verbe de la comparative présente une réalisation syntagmatique (canonique) de l'objet, la subordonnée doit être réalisée en P2, sous peine, au mieux, de recevoir une interprétation causale, au pire, d'être inacceptable. Entre ces deux pôles d'acceptabilité, il faut reconnaître une certaine « zone d'indécidabilité »".

\begin{tabular}{|l|l|l|l|||}
\hline (18) & a. & Jean aime Patrick comme Pierre aime Paul, c'est-à-dire pas beaucoup. & \\
\hline & b. & *Comme Pierre aime Paul, Jean aime Patrick, c'est-à-dire pas beaucoup. & \\
\hline \hline & c. & Comme Pierre aime Paul, Jean aime Patrick. & \\
\hline
\end{tabular}




\begin{tabular}{|c|c|}
\hline d. & Pierre parle de son potager, comme un pirate parlerait de son trésor. \\
\hline e. & ?Comme un pirate parlerait de son trésor, Pierre parle de son potager. \\
\hline$f$. & ${ }^{*}$ Comme il aurait traité son fils, il vous a traité. (cf. 6) \\
\hline
\end{tabular}

En (18a) l'interprétation comparative est limpide. Les difficultés d'interprétation pour $(18 \mathrm{~b}, \mathrm{e}, \mathrm{f})$ (même si (18e) est un peu meilleure pour certains ${ }^{10}$ ) proviennent peut-être d'une concurrence entre les deux constructions. Et, lorsqu'on supprime le reformulatif de (18b), on obtient clairement une interprétation causale (18c).

\subsubsection{Comparatives MOD $S$ et MOD GV, avec réalisation non canonique de l'objet}

14 Quand le verbe de la comparative présente une réalisation non canonique de l'objet, la subordonnée peut être réalisée en P1, P2, ou même en position incidente dans le cas d'un $\mathrm{S}$ modifié (souvent entre sujet et verbe). On entend par non canonique le fait que l'objet ne soit pas réalisé (c'est possible avec les verbes de discours) ou bien réalisé sous forme clitique.

\section{Position incidente} \begin{tabular}{l|l} 
(19) & $\begin{array}{l}\text { Le gouvernement, comme disait le ministre interrogé à l'instant, ne peut se permettre de céder aux } \\
\text { revendications. }\end{array}$
\end{tabular}

P1

\begin{tabular}{|l|l|l|}
\hline$(20)$ & a. & Comme l'eût fait un criminel amateur, il commit des fautes. \\
\hline \hline & b. & $\begin{array}{l}\text { Comme disait le ministre interrogé à l'instant, le gouvernement ne peut se permettre de céder } \\
\text { aux revendications. }\end{array}$ \\
\hline
\end{tabular}

P2

Il commit des fautes, comme l'eût fait un criminel amateur.

Le gouvernement ne peut se permettre de céder aux revendications, comme disait le ministre interrogé à l'instant.

15 Pour quelques locuteurs, l'antéposition de la comparative modifieur de GV est incertaine. Elle est majoritairement mauvaise lorsque l'objet est canoniquement réalisé (cf. §1.3.1.), et semble s'améliorer selon le degré d'anaphore (ou cataphore) que la subordonnée présente : taux d'acceptabilité correct avec reprise clitique d'un GN de la principale, taux d'acceptabilité important avec reprise par proforme verbale (faire ou le faire) du GV de la principale (20a). 


\subsubsection{Comparative MOD GVinf, avec réalisation non canonique de l'objet}

Pour les constituants modifiés autres que S -- soient GV, GVinf, GA et GN -- le patron P1, P2 doit être légèrement modifié dans la mesure où l'on examine la place de l'ajout devant ou derrière le constituant modifié, et non pas seulement devant ou derrière la principale. Ce type d'ajout a une portée locale, en général, la comparative ne peut se placer au-delà du constituant modifié (la contiguïté est requise). Nous appellerons donc $\mathrm{C} 1, \mathrm{C} 2$ les places possibles pour la subordonnée et le constituant modifié ${ }^{11}$. Les GV infinitifs modifiés ont valeur de GN (sujet ou compléments d'une préposition) ou sont compléments du V principal. Dans tous les cas, la subordonnée peut être en position incidente entre le Vinf et ses compléments. Lorsque le GVinf a valeur de GN sujet, l'ajout présente les trois possibilités : incidente (21a) ; C1 (21b) ; C2 (21c); mais étant donné sa portée locale, il ne peut être en P2 -- derrière la principale --, voir l'agrammaticalité de (21d,e).

\begin{tabular}{|l|l|l|l|}
\hline (21) & a. & $\begin{array}{l}\text { Utiliser, comme le font certains théâtres et surtout le Châtelet, soit l'Orchestre de Paris soit les } \\
\text { orchestres de la Radio est un détournement de mission et de subventions pour l'un et pour les } \\
\text { autres, dangereux pour l'existence même des orchestres de la Radio, formations destinées à } \\
\text { l'antenne, non aux fosses d'orchestre. (Le Monde) }\end{array}$ \\
\hline b. & $\begin{array}{l}\text { Comme le font certains théâtres et surtout le Châtelet, utiliser soit l'Orchestre de Paris soit les } \\
\text { orchestres de la Radio est un détournement de mission. }\end{array}$ \\
\hline c. & $\begin{array}{l}\text { Utiliser soit l'Orchestre de Paris soit les orchestres de la Radio, comme le font certains théâtres } \\
\text { et surtout le Châtelet, est un détournement de mission. }\end{array}$ \\
\hline d. & $\begin{array}{l}\text { *Utiliser soit l'Orchestre de Paris soit les orchestres de la Radio est un détournement de mission, } \\
\text { comme le font certains théâtres et surtout le Châtelet. }\end{array}$ \\
\hline e. & $\begin{array}{l}\text { *Revenir sur une limitation de sa souveraineté contrevient à l'ambition générale, comme entend } \\
\text { le faire le régime de Bagdad. }\end{array}$ \\
\hline
\end{tabular}

Une comparative modifieur d'un GVinf appartenant au GV principal peut être en position incidente (22a) ou en C2 (22b), mais engendre des difficultés d'interprétation ou des ambiguïtés $^{12}$ lorsqu'elle est en C1 $(23 a, b)$ : la portée de l'ajout est locale.

\begin{tabular}{|l|l|l|}
\hline (22) & a. & $\begin{array}{l}\text { La municipalité a interdit de peindre en rose, comme comptaient le faire mes voisins, les } \\
\text { barrières mitoyennes et les volets en façade. }\end{array}$ \\
\hline & b. & L'ONU contraint les soldats de l'Union à rebrousser chemin, comme ils le firent en 14-18. \\
\hline (23) & a. & $\begin{array}{l}\text { ?La municipalité a interdit, comme comptaient le faire mes voisins, de peindre en rose les } \\
\text { barrières mitoyennes et les volets en façade. }\end{array}$ \\
\hline & b. & ?L'ONU contraint les soldats de l'Union, comme ils le firent en 14-18, à rebrousser chemin. \\
\hline
\end{tabular}


Lorsque la comparative modifie un GVinf appartenant au GV principal (avec objet non canoniquement réalisé) et qu'on l'antépose à la phrase principale (P1), elle modifie alors le GV de cette dernière. Le faire reprend le GV principal entier, non pas le GVinf seul.

\begin{tabular}{|l|l|l|}
\hline (24) & a. & $\begin{array}{l}\text { Comme comptaient le faire mes voisins, la municipalité a interdit de peindre en rose les } \\
\text { barrières mitoyennes et les volets en façade. }\end{array}$ \\
\hline & b. & Comme ils le firent en 14-18, l'ONU a contraint les soldats de l'Union à rebrousser chemin. \\
\hline
\end{tabular}

19 On n'observe pas de comparative modifieur d'un GV infinitif présentant une réalisation canonique de l'objet direct. Dès que l'on tente d'en construire, on obtient une interprétation causale (25a), ou l'on éprouve les mêmes difficultés d'interprétation qu'au \$1.3.1, et ce, quelle que soit sa place par rapport au constituant modifié :

\section{C1 (possible interprétation causale)}

\begin{tabular}{|l|l|l|l|}
\hline (25) & a. & $\begin{array}{l}\text { ?Comme le ministère de la Santé a eu coutume de dépenser aléatoirement les bénéfices de la } \\
\text { Sécurité sociale, dilapider les fonds publics ne fera qu'aggraver l'équilibre déjà incertain du } \\
\text { budget de l'état. }\end{array}$
\end{tabular}

C2

*Dilapider les fonds publics, comme le ministère de la Santé a eu coutume de dépenser aléatoirement les bénéfices de la Sécurité sociale, ne fera qu'aggraver l'équilibre déjà incertain du budget de l'état.

P2

*Dilapider les fonds publics ne fera qu'aggraver l'équilibre déjà incertain du budget de l'état, comme le ministère de la Santé a eu coutume de dépenser aléatoirement les bénéfices de la Sécurité sociale.

\section{Position incidente}

d. *Dilapider, comme le ministère de la Santé a eu coutume de dépenser ses bénéfices, les fonds publics et les revenus indirects, ne fera qu'aggraver l'équilibre déjà incertain du budget de l'état. 


\subsubsection{Comparatives MOD A ou MOD GA, avec réalisation non canonique de l'objet}

Les comparatives modifieur de A ou GA ne peuvent être ni en P1 $(26 a)^{13}$, ni en C1 (26b), mais la position incidente est disponible entre le A et ses compléments, s'ils sont suffisamment lourds (26c). C2 est la place la plus fréquente (26d).

P1

\begin{tabular}{|l|l|l|l}
\hline (26) & a. & *Comme peut l'être un fils de notable, il est fier. \\
\hline
\end{tabular}

C1

*Il est, comme peut l'être un fils de notable, fier.

\section{Position incidente}

Il est très fier, comme peut l'être un fils de notable, de ses ancêtres qui ont conquis l'Amérique.

C2

Il est très fier de ses ancêtres, comme peut l'être un fils de notable.

21 C2 peut ne pas être possible si le GA est lourd et si le GV de l'ajout ne réalise pas de complément (27b), auquel cas la position incidente est requise (27a).

\begin{tabular}{|l|l|l|}
\hline (27) & a. & Il s'en va bon train, heureux comme il est d'avoir réussi son examen. \\
\hline \hline & b. & *Il s'en va bon train, heureux d'avoir réussi son examen comme il est. \\
\hline
\end{tabular}

\subsubsection{Comparatives MOD GN, avec réalisation non canonique de l'objet}

Le GN modifié ne semble pas tolérer d'insertion (28a). L'ajout ne peut être ni en C1 $(28 b, c)$, ni en P1 (antéposition de la subordonnée à la principale, $(28 \mathrm{~d}, \mathrm{e}))$ : il est obligatoirement en $\mathrm{C} 2$, derrière le constituant modifié.

\section{Position incidente}

\begin{tabular}{|l|l|l|l|}
\hline$(28)$ & a. & *Je rêve à des gâteaux, comme ma nounou les préparait, au chocolat. \\
\hline
\end{tabular} 
C1

*Je mange, comme ma nounou les préparait, des gâteaux au chocolat.

? ?D'autre part, vous négligez la dimension historique, comme disait Mme de Staël, la "dignité du temps ", qui seule dessine l'évidence des progrès et minimise la portée des accidents de parcours.

En (28c) la portée locale de l'ajout porte sur dimension historique et non plus sur dignité du temps (cf. 11).

P1

*Comme ma nounou les préparait, je rêve à des gâteaux au chocolat.

*Comme l'avait baptisée George Bush le jour de son investiture, le 20 janvier 1989, La «maison du peuple » a subi ses derniers grands travaux sous la présidence du démocrate John Kennedy.

C2

\begin{tabular}{|l|l|l|}
\hline (29) & a. & Je rêve à des gâteaux au chocolat comme ma nounou les préparait. \\
\hline & b. & $\begin{array}{l}\text { La «maison du peuple ", comme l'avait baptisée George Bush le jour de son investiture, le } 20 \\
\text { janvier 1989, a subi ses derniers grands travaux sous la présidence du démocrate John Kennedy. }\end{array}$ \\
\hline
\end{tabular}

Il existe deux usages de la comparative modifieur de GN : la comparaison et l'incidente de discours ${ }^{14}$. Les incidentes de discours ont des propriétés pragmatiques particulières, sur lesquelles nous reviendrons (\$2). Un de leurs effets est de mettre en mention n'importe quelle partie d'un énoncé, quelle que soit sa fonction; autrement dit, tout GN de la principale est susceptible d'être suivi d'une incidente en comme.

\subsection{Bilan de la distribution}

Nous rappelons que les positions incidentes étudiées sont des insertions de syntagme, généralement signalées par des virgules, entre un constituant tête et ses compléments. Lorsque l'insertion se situe entre le sujet et le verbe, le sujet ne peut être clitique. Les positions frontières, gauche ou droite, sont appelées P1/P2 lorsqu'on examine la place de l'ajout par rapport au S principal, et sont appelées $\mathrm{C} 1 / \mathrm{C} 2$ quand elles correspondent aux frontières gauche ou droite d'un constituant modifié interne au $\mathrm{S}$ principal. Il arrive que $\mathrm{C} 1$ et $\mathrm{P} 1$ se confondent lorsque l'ajout modifie le GN sujet de la principale, ainsi que C2 et P2 dans le cas de la modification du GV, ou d'un GVinf complément du GV principal. 
Tableau 1 : Place de la construction causale et de la construction comparative MOD S objet non canonique

\begin{tabular}{|l|l|l|l|l|}
\hline \multicolumn{2}{|l|}{ Ajout causal MOD S } & \multicolumn{3}{l|}{$\begin{array}{l}\text { Ajout comparatif MOD S } \\
\text { obj. ncan }\end{array}$} \\
\hline P1 & + & P1 & + \\
\hline \hline P2 & - & P2 & + \\
\hline Position incidente & 16 & + & Position incidente & + \\
\hline
\end{tabular}

Tableau 2 : Distribution des places des constructions comparatives MOD GV et MOD GVinf

\begin{tabular}{|c|c|c|c|c|c|}
\hline & $\begin{array}{l}\text { MOD GV, } \\
\text { obj. ncan }\end{array}$ & $\begin{array}{l}\text { MOD GV, } \\
\text { obj. can }\end{array}$ & $\begin{array}{l}\text { MOD GVinf } \\
\text { sujet, } \\
\text { obj. ncan }\end{array}$ & $\begin{array}{l}\text { MOD GVinf } \\
\text { complément du V } \\
\text { principal, obj. ncan }\end{array}$ & $\begin{array}{l}\text { MOD } \\
\text { GVinf, } \\
\text { obj. can }\end{array}$ \\
\hline $\begin{array}{l}\mathrm{C} 1 \\
\text { exemples }\end{array}$ & $++^{17}$ & $+{ }^{18}$ & $\begin{array}{l}+ \\
(21 b)\end{array}$ & $\begin{array}{l}- \\
(23 a, b)\end{array}$ & $\mid \begin{array}{l}- \\
(25 a)\end{array}$ \\
\hline $\begin{array}{l}\text { C2 } \\
\text { exemples }\end{array}$ & $\mid \begin{array}{l}+ \\
(20 c)\end{array}$ & $\begin{array}{l}+ \\
(18 \mathrm{~d})\end{array}$ & $\begin{array}{l}+ \\
(21 c)\end{array}$ & $\begin{array}{l}+ \\
(22 b)\end{array}$ & $\begin{array}{l}- \\
(25 b)\end{array}$ \\
\hline $\begin{array}{l}\text { Position } \\
\text { incidente }\end{array}$ & $++^{19}$ & -20 & $\begin{array}{l}+ \\
(21 a)\end{array}$ & $\begin{array}{l}+ \\
(22 a)\end{array}$ & (25d) \\
\hline $\begin{array}{l}\text { P1 } \\
\text { exemples }\end{array}$ & $\begin{array}{l}+ \\
(20 a)\end{array}$ & $\begin{array}{l}- \\
(18 \mathrm{e}, \mathrm{f})\end{array}$ & $\begin{array}{l}+ \\
(21 b)\end{array}$ & $\begin{array}{l}- \\
(24 a, b)\end{array}$ & $\begin{array}{l}- \\
(25 a)\end{array}$ \\
\hline $\begin{array}{l}\text { P2 } \\
\text { exemples }\end{array}$ & $\mid \begin{array}{l}+ \\
(20 c)\end{array}$ & + & $\begin{array}{l}- \\
(21 d)\end{array}$ & $\begin{array}{l}+ \\
(22 b)\end{array}$ & \begin{tabular}{|l}
- \\
$(25 c)$
\end{tabular} \\
\hline
\end{tabular}

La portée locale de l'ajout comparatif obj. ncan MOD SYNTAGME (autre que S principal) a plusieurs conséquences : l'ajout MOD GVinf (interne au GV principal) ne peut se situer en C1 entre le V principal et le GVinf (23a,b), ni en P1 (c'est-à-dire avant le GV auquel il appartient $(24 a, b))$, sous peine de modifier non pas le GVinf mais le V ou le GV principal tout entier. De même, l'ajout MOD GVinf (sujet) ne peut être au-delà du sujet, en P2 (autrement dit en fin de phrase). Lorsque l'ajout MOD GV est obj. can, son interprétation entre en concurrence avec celle de l'ajout causal s'il se situe en P1. La position incidente ne semble pas possible, mais C1 l'est à condition que le syntagme GV qui suit l'ajout soit lourd $^{21}$. 
Tableau 3 : Distribution des places des constructions comparatives MOD GN et MOD GA

\begin{tabular}{|l|l|l|l|l|l|}
\hline & $\begin{array}{l}\text { C1 } \\
\text { exemples }\end{array}$ & $\begin{array}{l}\text { exemples } \\
\text { exemples }\end{array}$ & $\begin{array}{l}\text { Position incidente } \\
\text { exemples }\end{array}$ & $\begin{array}{l}\text { P2 } \\
\text { exemples }\end{array}$ \\
\hline MOD GN & - & + & - & - & + \\
& $(28 \mathrm{~b}, \mathrm{c})$ & $(29 \mathrm{a}, \mathrm{b})$ & $(28 \mathrm{a})$ & $(28 \mathrm{~d}, \mathrm{e})$ & $(29 \mathrm{a}, \mathrm{b})$ \\
\hline MOD GA & - & + & + & - & + \\
& $(26 \mathrm{~b})$ & $(26 \mathrm{~d})$ & $(26 \mathrm{c})$ & $(26 \mathrm{a})$ & $(26 \mathrm{~d})$ \\
\hline
\end{tabular}

Les contraintes sur la réalisation linéaire de ces ajouts sont une composante entrant dans leur description grammaticale. La place de l'ajout causal est fixe (P1) (excepté les positions d'insertion courantes dans le domaine $S$ ) et ne dépend d'aucune autre contrainte. Celle des ajouts comparatifs est déterminée par le constituant modifié et par une contrainte interne sur la réalisation de l'objet, mais une régularité se dégage : tout ajout comparatif peut être en C2, à droite du constituant modifié. Lorsque le verbe de la subordonnée présente un objet non canonique, l'ajout peut être déplaçable, à moins que la contrainte sur sa portée locale ou sur le poids des syntagmes ne l'en empêche.

\subsection{Corollaire à la réalisation non syntagmatique de l'objet : l'inversion du sujet en comparative}

28 La comparative est caractérisée par une fréquente réalisation non canonique de l'objet. Ce trait apparaît très souvent avec une réalisation non canonique de l'ordre linéaire des syntagmes de la subordonnée. En fait, l'inversion du sujet est structuralement conditionnée par la forme de l'objet.

29 A proprement parler, la construction causale ne déclenche pas d'inversion du sujet. Si l'on rencontre parfois de telles réalisations introduites par comme causal, c'est qu'il s'agit de constructions avec inversion existant par ailleurs en phrase radicale ${ }^{22}$.

\begin{tabular}{|l|l|l|}
\hline (30) & a. & Comme n'était jamais survenu aucun événement de ce genre (...) \\
\hline & b. & Comme à la gloire succède la misère, méfions-nous! \\
\hline & c. & Comme sur la place se dresse la cathédrale, tu pourras te repérer. \\
\hline & d. & Comme interviennent soudain les forces de l'ordre, les manifestants se dispersent. \\
\hline
\end{tabular}

$\mathrm{Au}$ contraire, l'inversion du sujet est très fréquente en comparatives. Elle présente les mêmes conditions de réalisation que dans un contexte QU-, ce qu'on nomme « inversion stylistique ». Etant donnée l'impossibilité de la séquence ${ }^{23}$ *V-GN[Préd.-]-GN (où GN [Préd.-] est un GN non prédicatif), l'apparition d'un sujet postposé est fonction de la forme non canonique de l'objet (en contexte QU- l'objet est relativisé). En comparative, l'objet non canonique n'est pas réalisé (proforme verbale, verbes de discours), est 
inexistant (V lexicalement intransitifs), ou bien il est de forme clitique (clitique de la proforme verbale ou clitique référentiel).

31 L'inversion QU- n'est que très rarement obligatoire, par exemple dans le cas où être et faire sont employés avec un seul complément (sans ajout ni complément autres que l'objet ou l'attribut relativisé) :

\begin{tabular}{|l|l|l|}
\hline (31) & a. & *Les dessins que les enfants font \\
\hline \hline & b. & *L'homme que Paul est \\
\hline
\end{tabular}

En comparative, on observe le même phénomène. On constate la distribution suivante : lorsque le verbe est faire, une copule ou un verbe lexicalement intransitif, qu'il est conjugué à un temps simple (surtout au présent de l'indicatif) ou n'est pas complément d'un verbe, on est obligé de réaliser un constituant à sa droite : un sujet inversé ou un ajout (de type circonstanciel).

\begin{tabular}{|l|l|l|}
\hline (32) & a. & Max grignote toute la journée des friandises comme les gens inactifs font souvent. \\
\hline \hline & b. & *Max grignote toute la journée des friandises comme les gens inactifs font. \\
\hline$(33$ & a. & Poucette était née comme viennent les roses, un matin de printemps. \\
\hline \hline & b. & ?Poucette était née comme les roses viennent, un matin de printemps. \\
\hline & c. & *Poucette était née un matin de printemps, comme les roses viennent. \\
\hline$(34)$ & & *Il ment tout le temps comme un enfant ferait. \\
\hline$(35)$ & a. & Son argent disparaît comme la neige fond au soleil. \\
\hline \hline & b. & *Son argent disparaît comme la neige fond. \\
\hline & c. & Son argent disparaît comme fond la neige. \\
\hline \hline$(36)$ & a. & Malade comme était la corvette, elle était peu résistante aux secousses. \\
\hline & b. & *Malade comme la corvette était, elle était peu résistante aux secousses. \\
\hline
\end{tabular}

33 Cette contrainte est ténue, mais semble-t-il inviolable. Elle appartient aux propriétés lexicales d'une classe de verbes hétérogène, dépend de la construction dans laquelle ils entrent (propriété syntaxique ${ }^{24}$ ), et a des impératifs sur les temps (contrainte morphologique). Il se peut également que le niveau phonologique intervienne, sans qu'on puisse rien prouver ici ; de tout façon, une fois de plus, les problèmes de représentation se posent.

Dans le cas où faire et être sont construits avec un clitique, la contrainte tombe, et l'on peut ne faire suivre le verbe par aucun élément. 


\begin{tabular}{|l|l|l|}
\hline (37) & a. & Il est fier comme un fils de notable peut l'être. \\
\hline \hline & b. & Il grignote toute la journée des friandises comme les gens inactifs le font. \\
\hline & c. & Il ment tout le temps comme un enfant le ferait. \\
\hline
\end{tabular}

\section{Traitement de l'information en construction comparative : phénomènes de copie et d'ellipse}

Sans entrer dans l'analyse sémantique approfondie de ces constructions, nous décrivons ici les stratégies de langue utilisées dans l'expression de la comparaison. La comparaison en comme est une comparaison qualitative, il s'agit d'une mise en conformité de deux information $\mathrm{s}^{25}$. Elle met en présence des relations d'identification, au sens large, entre les arguments ou entre les procès de ces arguments. La caractérisation du comparant (contenue dans l'ajout) est variable, mais comporte toujours au moins une information nouvelle. Cette dernière se réalise par du matériel lexical neuf, et concerne au moins un des éléments suivants : l'argument sujet, objet, ou le procès de l'ajout comparatif. S'il y a mise en conformité d'une information donnée avec une information nouvelle, il y a parallèlement une relation entre les informations qui sont redondantes. Dans l'ajout, ces dernières sont l'objet d'une copie lexicale (répétition d'un item), d'une proforme (nominale ou verbale), ou d'une ellipse (syntaxique ou lexicale).

Prenons le cas d'une modification de GV. On peut observer un changement lexical pour l'argument sujet et le prédicat (38a), pour les arguments sujet et objet (38b), pour le prédicat et l'objet (38c), ou pour l'un des deux arguments sujet ou objet $(38 \mathrm{~d}, \mathrm{e})$ :

\begin{tabular}{|l|l|l|l|}
\hline (38) & a. & Ces jeunes femmes dansaient comme couraient les déesses de l'antiquité. \\
\hline \hline & b. & Jean parle de son jardin comme un pirate parlerait de son trésor. \\
\hline & c. & Mon voisin parle à ses enfants comme il rouspète après son chien. \\
\hline & d. & Jean parle de son jardin comme en parlerait un poète. \\
\hline & e. & Jean parle de son jardin comme il parlerait d'un trésor. \\
\hline & f. & Jean parle de son jardin comme le ferait un poète. \\
\hline
\end{tabular}

Parallèlement on remarque la copie des informations redondantes: copie lexicale du prédicat (copie du V ou du GV de la principale, avec changement de temps possible, exemples $(38 \mathrm{~b}, \mathrm{~d}, \mathrm{e}))$, anaphore de l'argument sujet $(38 \mathrm{c}, \mathrm{e})$, objet $(38 \mathrm{~d})$, ou anaphore verbale (38f).

Mais l'information redondante peut également être traitée par une absence: absence lexicale via l'ellipse de l'argument sujet et du prédicat (39a), du prédicat et de l'argument 
objet (39b) ou du prédicat seul (39c); absence syntaxique (39d) lorsqu'en MOD GN, par exemple, le verbe de la subordonnée ne réalise pas son objet et qu'il est lié au GN modifié :

\begin{tabular}{|l|l|l|}
\hline (39) & a. & Paul parle à ses enfants comme à son chien. \\
\hline \hline & b. & Max grignote des friandises toute la journée comme un enfant. \\
\hline & c. & Le vent pénétrait son corps comme le soleil cette eau. \\
\hline & d. & La « dignité du temps ", comme disait Mme de Staël, ne doit pas être oubliée. \\
\hline
\end{tabular}

Dans l'ajout comparatif on observe donc deux types de traitements de l'information redondante ou nouvelle: un traitement lexical, par changement ou suppression d'un item; et un traitement syntaxique par proforme ou dépendance à distance d'un argument. Nous choisissons d'étudier plus particulièrement les stratégies syntaxiques, puis d'aborder certaines caractéristiques de l'ellipse lexicale.

\subsection{Liage}

Les procédures de liage, essentiellement en jeu dans les comparatives, sont à dissocier de la procédure de modification, elles ne se situent pas au même niveau d'organisation, et relèvent d'un calcul interprétatif distinct. La modification participe de la macrostructure de la construction, c'est un trait obligatoire ; l'anaphore, elle, est un mécanisme de langue indépendant, ayant des contraintes propres ${ }^{26}$-- sa présence est un corollaire aux propriétés sémantiques de la construction comparative. Dans certaines des formes étudiées, les deux procédures peuvent se superposer, liant et modifiant parfois le même élément. Nous entendons ici par liage, le phénomène suivant :

Principe permettant d'associer (lier) référentiellement deux éléments appartenant à deux domaines phrastiques distincts. L'un des éléments est lexicalement réalisé, l'autre est une proforme nominale ou verbale, ou bien un argument qui se signale par une absence syntaxiquement remarquable ${ }^{27}$.

\subsubsection{Subordonnées causales et comparatives : reprise anaphorique}

41 Le domaine de la subordination est un terrain propice à l'anaphore puisqu'on a une succession de deux domaines phrastiques. Rien d'étonnant, donc, à ce qu'on en produise en construction causale ou en construction comparative, comme dans d'autres constructions subordonnées. Nous regardons principalement les marques d'anaphore complément en subordonnée, d'un constituant de la phrase principale.

\section{Anaphores en causale :}

On observe en (40) une anaphore (le faire) du GV principal, en (41) l'anaphore (clitique accusatif) d'un $\mathrm{GN}$ :

(40) Comme Pierre ne l'avait pas encore fait, il présenta sa femme à son nouvel employeur. 
(41) Comme Pierre ne l'avait pas vue, Marie en profita pour s'éclipser par la porte du salon.

\section{Anaphore du S principal en comparative :} anaphoriquement dans la subordonnée l'ensemble de la principale. L'élément référentiel lexicalement réalisé est donc, dans ces cas-là, le $\mathrm{S}$ entier.

\begin{tabular}{|l|l|ll|}
\hline (42) & a. & $\begin{array}{l}\text { Comme M. le président y faisait allusion tout à l'heure, les nouvelles dispositions } \\
\text { gouvernementales affaiblissent nos parts de marché. }\end{array}$ \\
\hline \hline & b. & $\begin{array}{l}\text { C'est le moyen d'éviter de laisser filer les déficits publics et, comme l'a remarqué M. Michel } \\
\text { Sapin, ministre français de l'économie et des finances, un engagement collectif dans ce sens } \\
\text { pourrait avoir une influence heureuse sur les décisions à venir de la Bundesbank en matière de } \\
\text { taux d'intérêt. (Le Monde) }\end{array}$ \\
\hline c. & $\begin{array}{l}\text { Pensez-vous, comme l'avancent les responsables de plusieurs organisations des Nations unies, } \\
\text { que l'oms a tendance à avoir une position hégémonique? (Le Monde) }\end{array}$ \\
\hline d. & $\begin{array}{l}\text { Je considère donc qu'il s'agit de travail clandestin et de placement payant, comme l'a relevé } \\
\text { l'inspection du travail. (Le Monde) }\end{array}$ \\
\hline
\end{tabular}

Ces comparatives, qui sont des incidentes de discours, sont très caractérisées. Elles sont toujours modifieurs de $\mathrm{S}$, et la subordonnée ne présente jamais d'objet canoniquement réalisé. On a une reprise clitique, ou un verbe de discours (dire) sans objet réalisé. Ici, anaphore et modification coïncident.

L'anaphore de S dans les subordonnées causales ne semble pas possible (43a), le cas échéant on obtient une interprétation comparative (43b) :

\begin{tabular}{|c|c|c|}
\hline (43) & a. & ${ }^{*}$ Comme nous $l_{i}^{\prime}$ avons remarqué, [il faisait beau. $]_{i}$ \\
\hline & & Comme Pierre nous $l e_{i}$ faisait remarquer, [il fo \\
\hline
\end{tabular}

\subsubsection{Stratégies de liage}

Il faut signaler que, dans les corpus consultés, les causales présentent généralement des compléments syntagmatiquement réalisés. Tous les exemples de causales avec anaphore ont été construits. En tant que propriété indépendante l'anaphore est possible, simplement, elle n'est pas fréquente. En ce qui concerne les constructions comparatives, en revanche, l'usage de l'anaphore est majoritaire. Nous étudions les trois procédés les plus rencontrés. 


\subsubsection{Clitique objet} anaphore de S) exemple (45a), adjointes au GV principal (avec anaphore de l'objet du V principal) exemples $(45 \mathrm{~b}, \mathrm{c})$, adjointes à un $\mathrm{GN}$ (anaphore du GN sujet ou objet de la principale) exemples ( $45 \mathrm{~d}, \mathrm{e})$, et adjointes à $\mathrm{A}$ (anaphore de l'adjectif attribut) exemple (45f).

\begin{tabular}{|c|c|c|}
\hline$(45)$ & a. & $\begin{array}{l}\text { Même si la France, comme l'affirme le Quai d'Orsay, n'a pas de réserves à exprimer sur cette } \\
\text { attaque d'un site nucléaire à Bagdad, il serait bon de se soucier de la population. }\end{array}$ \\
\hline & $b$. & $\begin{array}{l}\text { Je songe à des moulins beaux, forts et vigoureux, comme y songerait un enfant captivé par le } \\
\text { vent. }\end{array}$ \\
\hline & $c$. & Jules parle de son jardin comme un poète en parlerait. \\
\hline & $d$. & Je rêve à des gâteaux au chocolat comme ma nounou les préparait. \\
\hline & e. & $\begin{array}{l}\text { La «maison du peuple ", comme l'avait baptisée George Bush le jour de son investiture, le } 20 \\
\text { janvier 1989, a subi ses derniers grands travaux sous la présidence du démocrate John Kennedy. }\end{array}$ \\
\hline & $f$. & Il est fier comme peut l'être un fils de notable. \\
\hline
\end{tabular}

\subsubsection{Absence de réalisation du complément objet dans la subordonnée}

Nous abordons le cas de verbes transitifs ne réalisant pas leur objet dans la subordonnée. Ces verbes sont représentés comme ne réalisant pas de complément mais ils entretiennent une dépendance à distance permettant de saturer l'argument avec un élément du S principal. Cet argument peut correspondre au $\mathrm{S}$ entier, dans le cas des comparatives modifieur de $\mathrm{S}$, ou à un constituant de $\mathrm{S}$.

Le phénomène décrit provient ici de propriétés lexicales de certains verbes dits de discours, dont le représentant est le verbe dire. La classe de ces verbes est assez fluctuante, on l'élargirait volontiers à une classe des verbes "d'expression " sans pour autant en donner des critères sémantiques d'identification précis. Ils appartiennent à deux constructions prototypiques: l'incise et l'incidente de discours en comme. Ces constructions sont dotées de propriétés pragmatiques -- le discours rapporté --, syntaxiques et lexicales particulières ${ }^{28}$. En dehors de ces constructions, il est difficile de construire ces verbes de discours sans objet réalisé. En construction causale, par exemple, on est obligé de réaliser l'objet clitique : 


\begin{tabular}{|l|l|l|l|}
\hline (46) & a. & Comme il ne le lui avait pas encore dit, au matin Pierre déclara à sa femme qu'il était viré. \\
\hline & b. & *Comme il n'avait pas encore dit, au matin Pierre déclara à sa femme qu'il était viré. \\
\hline & c. & *Comme il ne t'avait pas encore dit, au matin Pierre te déclara qu'il était viré. \\
\hline
\end{tabular}

51 L'agrammaticalité de (46b) et (46c) montre que le liage à distance de l'argument objet des verbes de discours ne peut se réaliser en construction causale ${ }^{29}$.

52 En comparatives, les réalisations sont tout de même contraintes. L'argument à distance $\mathrm{du}$ verbe de discours ne peut être qu'une catégorie susceptible de recouvrer une « valeur » GN : soit S, GN ou GVinf. Autrement dit, ce ne peut être le GV de la principale, et lorsqu'il y a anaphore de ce GV, elle est réalisée via une proforme verbale (faire ou le faire). Les exemples suivants concernent le liage de l'objet non saturé du verbe de la subordonnée avec un S (47), un GVinf (48), un GN (49). Les comparatives sont respectivement adjointes au constituant lié, soit, à $\mathrm{S}$, à GVinf, et à GN.

\begin{tabular}{|l||l|}
\hline (47) & $\begin{array}{l}\text { Comme disait le ministre interrogé à l'instant, le gouvernement ne peut se permettre de céder aux } \\
\text { revendications. }\end{array}$ \\
\hline$(48)$ & Dévaluer notre monnaie, comme préconisent certains, ne servira pas l'économie déjà fragilisée. \\
\hline$(49)$ & $\begin{array}{l}\text { D'autre part, vous négligez la dimension historique, la « dignité du temps ", comme disait Mme de } \\
\text { Staël, qui seule dessine l'évidence des progrès et minimise la portée des accidents de parcours. }\end{array}$ \\
\hline
\end{tabular}

53 Il existe un second cas de non réalisation du complément du verbe de la subordonnée, celui des comparatives modifieur de GA. Le liage se fait entre l'argument non réalisé et l'adjectif modifié. Ici, on ne sait si l'on doit s'appuyer sur des propriétés lexicales du verbe copule, et plus généralement sur celle des verbes d'état, pour expliquer cette dépendance à distance. Toujours est-il qu'on est face à une construction très contrainte ( $\mathrm{C} 2$ quasi obligatoire), présentant des traits communs avec la comparative en que (emploi exclusif des verbes d'état, possibilité d'adjonction de l'ajout à $\mathrm{A}$, non à $\mathrm{GA}$ ) .

\begin{tabular}{|l|l|ll|}
\hline (50) & a. & Malade comme était la corvette, elle était peu résistante aux secousses. (Grevisse \$1085) \\
\hline & b. & Aussi belle qu'était la corvette, elle était peu résistante aux secousses. \\
\hline & c. & Il s'en va bon train, heureux comme il est d'avoir réussi son examen. \\
\hline \hline & d. & Il s'en va bon train, si heureux qu'il est d'avoir réussi son examen. \\
\hline
\end{tabular}

54 Mais, à la différence des comparatives incidentes de discours, l'adjectif peut être réalisé, c'est-à-dire en l'occurrence, copié :

\begin{tabular}{|l|l|l}
\hline (51) & a. & Il est fier comme est fier un père venant de marier sa fille.
\end{tabular} 


\begin{tabular}{|l|l|l|}
\hline & b. & ?Il est aussi fier qu'est fier un père venant de marier sa fille. \\
\hline \hline & & Par ailleurs, on peut également réaliser l'adjectif sous forme clitique: \\
\hline$(52)$ & a. & Il est fier comme peut l'être un fils de notable. \\
\hline & b. & Il est aussi fier que peut l'être un fils de notable. \\
\hline
\end{tabular}

\subsubsection{Proforme verbale}

L'utilisation de la proforme verbale faire ou le faire est très importante dans les constructions comparatives. Là encore, bien qu'il s'agisse d'une propriété indépendante, que l'on peut rencontrer dans d'autres constructions (comme en causale, exemple (53)), il semble que la comparaison constitue un cadre de réalisation privilégié.

(53) Comme Pierre ne l'avait pas encore fait, il présenta sa femme à son nouvel employeur.

La proforme a des propriétés propres, qui en font une entrée lexicale à part entière. Tout d'abord, l'objet n'est jamais canoniquement réalisé, soit il est absent, soit il est clitique. Deuxièmement, cet objet n'est pas lié à un élément de la phrase matrice; qu'il soit clitique ou absent, aucun élément de la phrase matrice ne peut être lexicalement restitué en ses place et fonction ${ }^{30}$. Troisièmement, faire ou le faire reprend l'ensemble du contenu du GV principal ou d'un GVinf : Verbe + compléments.

Le clitique n'est pas référentiel ${ }^{31}$, il a un rôle essentiellement morphologique, permettant de discriminer l'emploi proforme de faire, mais permet également de valider l'hypothèse selon laquelle on a une structure transitive du verbe (non intransitive). On le dit optionnel (Grevisse \$745b), pourtant on note des variations d'acceptabilité selon qu'on le réalise ou non dans certains énoncés ${ }^{32}$. Employé avec faire proforme, il est neutre (forme invariable, masculin singulier).

\begin{tabular}{|l|l|l|}
\hline (54) & a. & Il parle de son jardin comme ferait un poète. \\
\hline & b. & Il parle de son jardin comme le ferait un poète. \\
\hline \hline & c. & *Il parle de son jardin comme en ferait un poète. \\
\hline
\end{tabular}

Ici, en (54a) et (54b), les acceptabilités ne varient pas, la présence du clitique en (54b) ne change pas le sens. Le couple $(54 \mathrm{~b}, \mathrm{c})$ montre que la morphologie du clitique de la proforme ne correspond pas à l'anaphore du complément du verbe principal. Il n'est pas lexicalement restituable auprès de faire sans changer la sous-catégorisation du verbe (voir ci-dessous Deuxième cas -- faire GN de GN) :

(55) *comme ferait de son jardin un poète 
Premier cas : Proforme, structure de type transitif; exemples avec comparatives adjointes à GV, ou à GVinf :

\begin{tabular}{|l|l|l|}
\hline (56) & a. & †Max grignote toute la journée des friandises comme le font souvent les gens inactifs. \\
\hline & b. & $\begin{array}{l}\text { Fabrice n'eut aucun effort à faire pour se conduire comme l'eût fait Fénelon en pareille } \\
\text { occurrence. (Grevisse) }\end{array}$ \\
\hline & c. & $\begin{array}{l}\text { L'ambition générale est de contrer toute velléité de l'Irak de revenir sur cette limitation de sa } \\
\text { souveraineté, comme entend le faire le régime de Bagdad en posant des conditions aux avions } \\
\text { des inspecteurs du désarmement de l'ONU qui survoleraient cette zone d'exclusion. (Le Monde) }\end{array}$ \\
\hline
\end{tabular}

\section{Deuxième cas : Proforme, structure de type ditransitif}

Faire ou le faire reprend l'ensemble du GV principal mais présente une sous-catégorisation ditransitive dans laquelle seul le second complément, de catégorie GP, est syntagmatiquement réalisé. Le schéma argumental du verbe est alors paraphrasable en : faire quelque chose de/devant/pour/avec, etc. quelqu'un. On rapporte ici quelques uns des nombreux exemples cités par Grevisse $(\$ 745 b)^{33}$ :

\begin{tabular}{|l|l|l|}
\hline (57) & a. & Regardant du coin de l'œil l'entaille comme il eût fait d'une bête aux aguets ${ }^{34}$. \\
\hline & b. & Jamais je n'avais regardé, détaillé un être humain comme je le faisais de celui-ci. \\
\hline \hline & c. & On ne me grondait pas comme on l'eût fait pour les autres. \\
\hline
\end{tabular}

\section{Troisième cas : Ambiguïté possible entre proforme et usage standard de faire}

Dans certains énoncés, selon l'analyse du clitique du verbe faire, on obtient deux constructions différentes. Ou bien on est face à une comparative modifieur de GN avec anaphore du GN (dans ces cas-là on a un emploi «standard » de faire -- proche de produire, fabriquer, etc.), ou bien on se trouve dans un cas de MOD GV avec reprise par proforme verbale. Regardons les exemples suivants :

\begin{tabular}{|l|l|l}
\hline (58) & a. & Il fit des fautes comme eut fait un criminel amateur.
\end{tabular} 


\begin{tabular}{|l|l|l|}
\hline & b. & *Il fit des fautes comme eût commis un criminel amateur. \\
\hline \hline & c. & Il fit des fautes comme l'eût fait un criminel amateur. \\
\hline & d. & Il fit des fautes comme en eût fait un criminel amateur. \\
\hline
\end{tabular}

En (58b), on a substitué le verbe commettre au verbe faire de (58a), dont la souscatégorisation est semblable et le sens proche. L'agrammaticalité de (58b) montre qu'en (58a) on n'a pas affaire au synonyme de commettre (emploi standard de faire), mais à la proforme. Dans ce contexte commettre ne peut être employé sans complément (clitique en quantitatif ou réalisation syntagmatique eût commit des fautes). Pour maintenir l'interprétation MOD GV, faire proforme doit être employé sans complément (exemple (58a)) ou avec le clitique neutre (exemple (58c)); mais il ne peut présenter de clitique référentiel. Dans le cas où faire présente un clitique morphologiquement et référentiellement adéquat à un constituant nominal de la principale (avec accord en genre, nombre et correspondance morphologique avec la forme GP/GN du constituant), on est face à un emploi standard du verbe faire, non à la proforme. L'ajout modifie alors ce constituant nominal, non plus le GV principal.

\begin{tabular}{|l|l|l|l|}
\hline (59) & a. & Je fabrique des cerfs-volants comme le ferait un enfant passionné par le vent. \\
\hline \hline & b. & Je fabrique des cerfs-volants comme les font les artisans japonais. \\
\hline & c. & Je rêve à des gâteaux au chocolat comme les faisait ma grand-mère. \\
\hline & d. & Je rêve à des gâteaux au chocolat comme en faisait ma grand-mère. \\
\hline & e. & Une tourte à l'endive, comme la font les ménagères du Cantal, c'est extraordinaire! \\
\hline
\end{tabular}

La variation du clitique en (59a) et (59b) montre une variation du constituant modifié. Conséquence logique de la reprise d'un constituant nominal, la modification de GN s'applique quelle que soit sa fonction dans le S principal (GN objet pluriel en (59c) et (59d), GN sujet fém. sing. en (59e)).

\subsubsection{Résultats}

Les procédés d'anaphore clitique et par proforme verbale sont indépendants des constructions dans lesquelles ils apparaissent. On les observe en constructions comparatives, causales, comme dans d'autres constructions subordonnées. Mais, au regard des corpus examinés, on confirme l'hypothèse selon laquelle les stratégies de liage observées dans les comparatives sont une conséquence des mécanismes sémantiques de la construction.

On a vu que la construction causale, modifieur de $\mathrm{S}$, pouvait présenter un liage via proformes clitique ou verbale, mais qu'il n'y avait pas de dépendance à distance de l'argument objet, ni de reprise possible de l'ensemble du S principal. A contrario, une des formes caractéristiques des comparatives, l'incidente de discours, autorise une reprise du constituant $\mathrm{S}$ modifié, via proforme clitique ou dépendance à distance d'un argument. Et 
c'est, semble-t-il, une caractéristique définitoire de cette construction. On résume l'ensemble des procédés d'anaphore en comparatives étudiés dans le tableau suivant:

\subsection{Ellipses lexicales en comparatives}

Comme causal, temporel et comme incidente de discours introduisent obligatoirement un syntagme phrastique. Comme comparatif peut être suivi par des séquences syntagmatiques très variées, mais les mécanismes sémantiques de la construction sont constants. Jusqu'à présent notre étude s'est portée sur les syntagmes phrastiques ; en se penchant sur le cas des ellipses dans ce contexte, on remarque que les différentes réalisations syntagmatiques à droite de comme comparatif ne changent pas le sens central de la construction. On fait donc l'hypothèse qu'on a affaire à la même construction.

\begin{tabular}{|l|l|l|}
\hline$(60)$ & a. & Max grignote des friandises toute la journée, comme ferait un enfant. \\
\hline \hline & b. & Max grignote des friandises toute la journée, comme un enfant. \\
\hline
\end{tabular}

En $(60 a, b)$ la présence ou l'absence du verbe de la subordonnée n'occasionne aucune différence, il s'agit d'une information redondante, n'affectant pas l'apport informationnel du comparant. Les remarques sont semblables en ce qui concerne une ellipse verbale avec réalisation des arguments sujet et objet :

\begin{tabular}{|l|l|l|}
\hline$(61)$ & a. & Mon âme attend le Seigneur comme les veilleurs l'aurore ${ }^{37}$. \\
\hline & b. & Mon âme attend le Seigneur comme les veilleurs attendent l'aurore. \\
\hline
\end{tabular}

C'est une caractéristique définitoire de l'ellipse verbale que d'être restituable dans tous les contextes où elle peut apparaître (cf. Zribi-Hertz (1986)). Les constructions en comme comparatif avec ellipse verbale dans l'ajout (cf. (61b)) ont été rapprochées de certains cas d'ellipses en coordination, ce qu'on a appelé construction à gapping (inter alia Zribi-Hertz (1986), Ross (1967)). La structure de type gapping a la forme suivante :

$\mathrm{GN}_{1}-\mathrm{GV}_{1}-\mathrm{X}$-conjonction- $\mathrm{GN}_{2}-\left(\mathrm{GV}_{2 \mathrm{e}}\right)-\mathrm{Y}$; où le second $\mathrm{GV}$ est en position vide.

71 (i) Le gapping ne se réalise que dans un contexte de coordination, les deux conjoints (clauses) sont au même niveau de constituance. Or dans le cas de MOD GV, l'ajout est adjoint au noeud GV, non pas au noeud S.

(ii) Dans le gapping, les compléments des verbes sont obligatoirement réalisés syntagmatiquement; dans la comparaison, on peut pronominaliser le complément $\mathrm{X}$ de $\mathrm{GV}_{1}$ :

\begin{tabular}{|l|l|l|}
\hline (62) & a. & Le soleil la pénétrait comme le soleil cette eau. \\
\hline & b. & {$\left[\right.$ Il y a des pommes et des poires dans la coupe à fruit ${ }^{*}$ Paul les mange et Marie les poires. } \\
\hline
\end{tabular}


73 (iii) Enfin, en gapping, le second conjoint doit impérativement être réalisé en seconde position, alors que l'ajout comparatif peut être antéposé :

\begin{tabular}{|l||l|l|}
\hline (63) & a. & *Marie les poires, et Paul mange les pommes. \\
\hline & b. & $\begin{array}{l}\text { Le monde social est parsemé de rappels à l'ordre qui ne fonctionnent comme tels que pour les } \\
\text { individus prédisposés à les apercevoir, et qui, comme le feu rouge le freinage, déclenchent des } \\
\text { dispositions corporelles. (Bourdieu, Méditations Pascaliennes : 20) }\end{array}$ \\
\hline
\end{tabular}

74 On obtient par là des arguments de taille pour différencier les constructions en comme du gapping.

\section{Conclusion}

Cette première étape descriptive des caractéristiques formelles des constructions causales et comparatives en comme nous a permis de mettre à jour leurs principales contraintes. Cet ensemble de propriétés doit être intégré dans la perspective d'une typologie des constructions du français, laquelle est une adaptation du projet de hiérarchie croisée des types de syntagmes élaboré par Sag (1997) en HPSG et un champ d'application pour la théorie des Grammaires de Constructions développée par Goldberg (1995).

76 Avant que de passer au stade de la formalisation, il sera nécessaire de résoudre quelques problèmes de représentation majeurs soulevés par la présente description.

77 Le premier problème concerne la réalisation linéaire de ces ajouts qui peut être différente, on l'a vu, de leur configuration syntaxique. Tout d'abord, caractéristique interne, il faut rendre compte du phénomène de l'inversion du sujet -- commun aux constructions QU- et aux comparatives -- de façon unifiée, en postulant une contrainte sur la réalisation non canonique de l'objet. Il est nécessaire pour cela de distinguer structure de constituance et réalisations linéaires. Si l'on se situe dans un modèle ne différenciant pas les deux niveaux, soit l'on multiplie le nombre des structures possibles, soit on doit avoir recours à des règles de transformation ${ }^{38}$.

Puis, caractéristique externe, se pose la question de la constituance de la construction face à la place de l'ajout. Le constituant modifié apparaît comme un paramètre déterminant la construction (causale ou comparative). Autrement dit, il est impératif d'indiquer dès la représentation déclarative de l'ajout le constituant soeur (constituant modifié), la relation de modification et les conséquences sur l'ordre linéaire. Chaque ajout sera prédéterminé à la modification de tel ou tel constituant. Le cas est semblable pour la représentation d'une modification via ajout relatif: on sait d'avance que la catégorie modifiée sera de type GN. C'est là une différence cruciale avec la représentation d'une relation de sous-catégorisation: un constituant n'est pas prédéterminé à être complément de tel autre.

79 Par ailleurs, il faut disposer d'un traitement de l'ordre linéaire, distinct de la constituance, capable d'ordonner les syntagmes les uns par rapport aux autres (qu'il s'agisse du constituant modifié et de l'ajout ou de la principale et de l'ajout), mais également capable d'exprimer les places d'insertion dans un syntagme. En résumé, le 
traitement linéaire de l'ensemble de la construction doit être sensible à des informations sémantiques sur l'ajout (cause ou comparaison), sur la constituance externe (élément modifié) et interne (réalisation de l'objet, et inversion possible du sujet), puisque chaque individu est obtenu par une combinatoire de contraintes.

Un deuxième ensemble de problèmes concerne la représentation des anaphores non nominales et des ellipses lexicales. Tout d'abord, il est nécessaire de disposer d'un module dédié au liage de façon autonome, permettant de co-indicer une proforme avec des éléments référentiels nominaux et non nominaux. Le cas se pose pour les anaphores d'adjectifs, il se pose également pour la représentation et le liage de la proforme verbale ( faire ou le faire). Lorsque l'argument non réalisé est lié à une catégorie $\mathrm{S}$, ou GVinf, susceptible de recouvrer une "valeur » de GN, une théorie générativiste avance qu'une position GN correspond à une fonction de GN. En HPSG, le liage, géré par le module sémantique, sélectionne les traits référentiels des éléments, mais ne se charge pas de rendre compte de leur propriétés de constituance.

81 En ce qui concerne les ellipses (lexicales) verbales, si l'on maintient une construction unique pour les réalisations avec et sans ellipse, le problème est bien entendu l'analyse de la constituance de l'ajout. D'une part, on sait que lorsque deux syntagmes apparaissent (l'argument sujet suivi de l'argument objet, comme en (62a)) aucune relation de dépendance ne les relie; d'autre part, si l'on conserve l'idée que la structure sémantique est maintenue, et qu'il y a bien ellipse verbale, comment peut-on traiter ces structures sans poser de "position vide "? Dans un modèle monostratal comme HPSG, dans lequel un syntagme phrastique est guidé par une tête verbale, et où l'on s'interdit de postuler des catégories non lexicalement réalisées, cela pose un véritable problème de représentation. Dans un modèle générativiste, dont les solutions requièrent le postulat de positions fonctionnelles vides, on avance que les phrases avec et sans ellipse ont une structure profonde identique. Nous proposons en HPSG que le module sémantique traite la constance interprétative observée. A bien y regarder, c'est le niveau qui offre le plus d'éléments explicatifs des variations formelles de ces constructions (en l'occurrence les stratégies de langue utilisées dans le traitement de l'information). Si le module sémantique prend en charge l'information sur les éléments ellipsés, que la construction est contrainte et typée comme développant une sémantique "comparative» (avec éléments identifiés, identifiants et caractérisations prédicatives), reste à définir comment cette information peut être transcrite en termes de représentation syntaxique ${ }^{39}$. Il y a une différence théorique de poids entre les deux parcours méthodologiques décrits. En prenant en compte de façon prioritaire le mécanisme interprétatif commun aux constructions comparatives en comme, on déclare que le niveau sémantique détermine ces réalisations syntaxiques. On obtient par là un plus haut degré de généralisation et on envisage de traiter non seulement les ajouts phrastiques (avec ou sans contenu prédicatif), mais aussi les ajouts non phrastiques (comme les exemples à «parangon», cf. Milner (1973), Il est bête comme ses pieds). Par conséquent, la caractérisation sémantique des constructions causales et comparatives en comme, qui n'était pas notre objet, mérite un travail à part entière, et peut être déterminant dans le traitement formel de ces constructions. 


\section{BIBLIOGRAPHIE}

ABEILLÉ, A., \& GODARD, D. (1997) French word order and lexical weight (à paraître).

BONAMI, O. \& GODARD, D. (1998) Inversion du sujet, constituance et ordre des mots (à paraître).

BOUMA, G., MALOUF, R. \& SAG, I. A. (1998) Satisfying Constraints on Extraction and Adjunction (à paraître).

CARON-PARGUE, J. (1992) «Connecteurs de subordination et opérations cognitives dans les verbalisations simultanées à une résolution de problème : si, parce que, comme, une fois que ", Travaux linguistiques du CERLICO 5, p. 198-220, Presses Universitaires de Rennes 2, Rennes.

CHOMSKY, N. (1980) « On Binding », Linguistic Inquiry 11-1.

DELABRE, M. (1984) « Les deux types de comparaison avec comme », Le français moderne 1984-1, p. 22-47.

DELAVEAU, A. \& DESMETS, M. (1998) « La Grammaire de constructions et le cas des phrases avec comme ", Séances du GDR 1171 Langage et connexionnisme.

DELÉCHELLE, G. (1989), L'Expression de la cause en anglais contemporain, ANRT, Lille.

DUPRIEZ, B. (1984) Gradus. Les procédés littéraires (dictionnaire), 10/18, C. Bourgois éditeur, Paris.

ERIKSSON, O. (1984) « Notes sur l'emploi de faire comme « verbum vicarium », Le Français moderne, avril 84, p. 48-60.

GOLDBERG, A. E. (1995) A Construction Grammar Approach To Argument Structure, Chicago Press.

GREVISSE, M. (1961) Le Bon Usage, 12è édition (1991), Editions Duculot, Paris.

GUIMIER, C. (1997) «Indices co-textuels et interprétation de as connecteur interpropositionnel », Co-texte et calcul du sens, Elsap-CNRS, Presses Universitaires de Caen, p. 165-180. GUIMIER, C. (1996) « Présence ou absence du sujet ou d'un complément essentiel dans certaines propositions introduites par as », Communication au Colloque du CERLICO 7-8 juin, Tours.

LE BIDOIS, R. (1950) L'inversion du sujet dans la prose contemporaine, Editions d'Artey, Paris.

LE GOFFIC, P. (1991) «Comme, adverbe connecteur intégratif : éléments pour une description », Travaux linguistiques du CERLICO 4, p. 11-31, Presses Universitaires de Rennes 2, Rennes.

MARANDIN, J.-M. (1997) Dans le titre se trouve le sujet. Ou : l'inversion locative en français, mémoire d'habilitation, Université Paris VII.

MILLER, P. (1992) Clitics and Constituents in Phrase Structure Grammar, Garland, New York.

MILNER, J.C. (1973) Arguments linguistiques, Mame, Paris.

MOIGNET, G. (1974) Etudes de psycho-systématique française, Klincksieck, Paris (sur le clitique de la proforme verbale voir p. 13-35).

MOUNIN, G. (1974) Dictionnaire de la linguistique, Collection Quadrige, PUF, Paris.

PINCHON, J. (1972) Les pronoms adverbiaux en et y, Droz, Genève (sur le clitique de la proforme verbale voir p. 168-188). 
REAPE, M. (1994) « Domain union and word-order variation in German », in J. Nerbonne, K. Netter et C. Pollard (éds.), German in Head-Driven Phrase Structure Grammar, p. 151-197, CSLI, Stanford.

SAG, I.A. (1997) « English relative clause constructions », Journal of Linguistics 33, p. 431-484.

SANDFELD, K. (1936) Syntaxe du Français Contemporain, II - Les propositions subordonnées, Droz, Genève.

REIS, M. (1984) « Pour une grammaire à trois valeurs : zone d'indécidabilité », DRLAV 31, p. 1-17.

TASSIN, G. (1998) L'ellipse dans la construction à gapping, mémoire de DEA en Sciences du langage, Université Paris X - Nanterre.

ZRIBI-HERTZ, A. (1986) Relations anaphoriques en français : esquisse d'une grammaire générative raisonnée de la réflexibilité et de l'ellipse structurale, Thèse de doctorat d'état, Université Paris VIII.

\section{NOTES}

1. Nous tenons à remercier A. Delaveau, K. Baschung, P. Gréa pour leur étroite collaboration et J. Anis, C. Normand, F. Kerleroux, M. Krazem, A. Kupsk, I.A. Sag, A. Tchobanov pour leurs remarques et conseils bibliographiques.

2. Un constituant tête d'une catégorie maximale est appelé «Branche-Tête » en HPSG (HEADDTR).

3. Nous utilisons l'étiquette du trait MODIFIEUR (MOD) du modèle HPSG comme abréviation.

4. Contra Milner (1973: 45, note 20).

5. Nous ne traitons pas des constructions en comme suivies d'une autre conjonction (comme si - S, comme quand - $\mathrm{S}$, etc.).

6. Et à la suite de Delaveau \& Desmets (1998).

7. Exception faite des propositions élaborées par Reape (1994), reprises dans Bonami \& Godard (1998), du principe « shuffle » en HPSG.

8. Observation confirmée par un travail sur corpus réalisé au cours du séminaire de DEA d'A. Delaveau (Paris X Nanterre : 1996-1998).

9. Voir Reis (1984).

10. Nous trouvons toutefois un (seul) exemple de subordonnée comparative en P1 avec objet canonique chez Sandfeld (\$256:425): Comme le voyageur arrivé enfin au terme de sa route aime à se souvenir des périls, des difficultés du voyage, Guillardin reprenait sa vie année par année.

11. Parfois, $\mathrm{C} 1$ correspond également à $\mathrm{P} 1$ lorsque l'ajout modifie le $\mathrm{GN}$ sujet, et $\mathrm{C} 2$ correspond à P2 lorsqu'il modifie le GN objet ou le GV.

12. Souvent, on hésite alors entre modification du GVinf et modification du GV principal tout entier. C1 semble toutefois possible dans le cas d'un GVinf complément de préposition: Pour, comme le font les grands auteurs, réussir une phrase courte, il faut beaucoup de mots.

13. (26a) semble s'améliorer si l'ajout modifie non plus un A mais un GA : ?Comme peut l'être un fils de notable, il est fier de ses ancêtres qui ont su s'illustrer dans la conquête de l'Amérique. Là encore, la question du poids des constituants entre en jeu.

14. Il faut, bien entendu, distinguer les incidentes de discours qui sont un type de construction (comprenant des propriétés pragmatiques et syntaxiques propres), de ce que nous appelons position incidente, qui correspond à une insertion syntagmatique entre la tête et les compléments d'un syntagme S, GV, GA.

15. obj. ncan : objet non canoniquement réalisé ; obj. can : objet canoniquement réalisé. 
16. Nous n'avons pas observé de réalisation incidente de l'ajout causal. Il semble néanmoins possible de l'insérer aux positions courantes de l'insertion dans un S, soit: entre le sujet (non clitique) et le verbe, entre le verbe et ses compléments. Par contre la position P2 (fin de phrase) provoque l'agrammaticalité de l'ensemble.

17. Les étudiants, comme ils le font chaque année, ont décidé de faire une fête à Noël.

18. Pierre, comme un pirate parlerait de son trésor, nous parle de son potager et de ses récoltes chaque fois qu'il le peut.

19. Jean parle, comme en parlerait un poète, du jardin dans lequel il a passé toute son enfance.

20. *Pierre nous parle, comme un pirate parlerait de son trésor, de son potager et de ses récoltes.

21. Sur la notion de poids des éléments syntaxiques voir Abeillé \& Godard (1997).

22. Se reporter à la typologie proposée dans Marandin (1997).

23. Nous nous appuyons sur la synthèse de Bonami \& Godard (1998).

24. C'est une propriété de la construction comparative et elle se traduit par une contrainte sur la constituance (ce doit être un verbe tête -- HEAD-DTR en HPSG)

25. Il s'agit d'une définition très générale mais commune aux grammaires traditionnelles (cf. Sandfeld §196 : 324) et aux travaux plus récents (cf. Delabre (1984), ou Guimier (1997)).

26. Sur les contraintes de l'anaphore voir Chomsky (1980).

27. Dans ce dernier cas, une théorie générativiste parle de catégorie vide, mais une grammaire syntagmatique de type HPSG peut résoudre cette absence en déclarant que le verbe n'est pas saturé pour tous ses compléments, tout en exprimant le liage des deux arguments référentiellement associés.

28. L'incise ne présente jamais d'objet réalisé (ni clitique, ni syntagmatique), n'a pas de marqueur initial, est toujours en P2, elle n'accepte pas la négation (alors que l'incidente en comme le fait), et l'inversion du sujet clitique ou GN est obligatoire (en incidente, pas d'inversion clitique, pas d'inversion obligatoire du GN sujet).

29. En (46a) le clitique objet est une anaphore de qu'il était viré non pas du S principal entier.

30. Dans une langue très littéraire on rencontre cependant faire réalisé avec à sa droite sujet et objet : comme fait son fuseau de lin la filandière (autres exemples dans Grevisse §745a). A noter que cet usage semble se maintenir au moins jusqu'au XVIIIème siècle (voir rem. historique 1 , au même §), ce qui indiquerait que l'interdiction d'une séquence ${ }^{*} \mathrm{~V}-\mathrm{GN}-\mathrm{GN}$ est relativement récente, ou bien que, contre toute attente, comme peut rendre licite une telle suite.

31. Sur le statut de ce clitique voir Miller (1992).

32. On ne s'engage pas ici sur la question de savoir si le et faire sont chacun dédiés à des tâches particulières dans l'anaphore, ou s'il s'agit d'un tout (sur ce point voir Grevisse (\$745b) citant Moignet \& Pinchon). Nous n'avons pas non plus entrepris un travail de corpus suffisant pour déterminer les raisons précises de cette variation d'acceptabilité, selon que le clitique est réalisé ou pas. A noter qu'en ancien français faire comme verbe substitut s'employait sans le.

33. Cf. Rem. Hist. 2 «L'utilisation d'une préposition daterait, selon Moignet, du début du XIXème siècle et serait contemporaine de l'introduction du pronom le ».

34. Dans l'exemple original (tiré de Malraux, Voie royale, II, 2), le marqueur de comparaison est ainsi que : ainsi qu'il eût fait d'une bête aux aguets ; cela ne change rien à notre analyse.

35. Exemple de GVinf repris par un clitique: Recourir à la force militaire, comme y songe déjà le président Clinton, déstabilisera l'équilibre politique mondial.

36. Exemple de GVinf lié à distance à l'objet du verbe de la subordonnée non réalisé : Recourir à la force militaire, comme disait le président Clinton l'autre jour, devient un leitmotiv aux assemblées de l'ONU

37. L'exemple original (Bible, trad. Crampon, Psaumes, CXXX) est : Mon âme attend le Seigneur plus que les veilleurs l'aurore.

38. Cela dit, même lorsqu'on fait la distinction entre ordre et constituance, comme en HPSG selon les propositions de Reape (1994) reprises dans Bonami \& Godard (1998), il faut encore spécifier la 
nature catégorielle des arguments réalisés (sans entrer en conflit avec les autres propriétés de la construction). En comparative, par exemple, l'adjectif attribut peut être réalisé en même temps que le sujet inversé : Il est fier comme est fier un père venant de marier sa fille; on aura donc une séquence $\mathrm{V}$-complément-sujet.

39. Il faudrait, par exemple, déclarer un trait SYNSEM verbal non-canonical pour les ellipses mais aussi pour la proforme verbale (faire, le faire).

\section{RÉSUMÉS}

Le présent article propose une étude descriptive des propriétés formelles des constructions phrastiques en comme causal et comparatif. Il met à jour les contraintes d'ordre linéaire attachées à la relation de modification déclenchée par ces ajouts, et rend compte des différentes stratégies utilisées pour le traitement de l'information en construction comparative (phénomènes de liage, ellipses, et copies lexicales). Elaborée dans la perspective d'une formalisation utltérieure en HPSG, cette étude soulève quelques points problématiques pour la représentation de ces données jusqu'alors peu étudiées.

This paper studies formal properties of causal and comparative comme clausal constructions. It points out word order constraints tied to the type of modification those adjuncts launched. It also examines different stategies used in the comparative constructions informational treatment (phenomena as binding, ellipsis, lexical copies). This study, developed in the pespective of a later HPSG fomalization, raises several problematic points for representing data harldy studied until then.

\section{AUTEUR}

\section{MARIANNE DESMETS}

